\title{
i-process Nucleosynthesis and Mass Retention Efficiency in He-shell Flash Evolution of Rapidly Accreting White Dwarfs
}

\author{
Pavel A. Denissenkov ${ }^{1,2,8}$, Falk Herwig ${ }^{1,2,8}$, Umberto Battino ${ }^{3,8}$, Christian Ritter ${ }^{1,2,8}$, Marco Pignatari ${ }^{4,5,8}$, Samuel Jones ${ }^{6,8}$, and \\ Bill Paxton ${ }^{7}$ \\ ${ }^{1}$ Department of Physics \& Astronomy, University of Victoria, P.O. Box 1700, STN CSC, Victoria, BC V8W 2Y2, Canada \\ 2 Joint Institute for Nuclear Astrophysics, Center for the Evolution of the Elements, Michigan State University, 640 South Shaw Lane, East Lansing, MI 48824, USA \\ ${ }^{3}$ Department of Physics, University of Basel, Klingelbergstrasse 82, CH-4056 Basel, Switzerland \\ ${ }^{4}$ E.A. Milne Centre for Astrophysics, Department of Physics \& Mathematics, University of Hull, Hull HU6 7RX, UK \\ ${ }^{5}$ Konkoly Observatory, Research Centre for Astronomy and Earth Sciences, Hungarian Academy of Sciences, Budapest, \\ Konkoly Thege Miklós út 15-17, 1121 Budapest, Hungary \\ ${ }^{6}$ Heidelberg Institute for Theoretical Studies, Schloss-Wolfsbrunnenweg 35, D-69118 Heidelberg, Germany \\ ${ }^{7}$ Kavli Institute for Theoretical Physics and Department of Physics, Kohn Hall, University of California, Santa Barbara, CA 93106, USA \\ Received 2016 October 26; revised 2016 December 9; accepted 2016 December 9; published 2017 January 9
}

\begin{abstract}
Based on stellar evolution simulations, we demonstrate that rapidly accreting white dwarfs (WDs) in close binary systems are an astrophysical site for the intermediate neutron-capture process. During recurrent and very strong $\mathrm{He}$-shell flashes in the stable H-burning accretion regime H-rich material enters the He-shell flash convection zone. ${ }^{12} \mathrm{C}(p, \gamma){ }^{13} \mathrm{~N}$ reactions release enough energy to potentially impact convection, and i process is activated through the ${ }^{13} \mathrm{C}(\alpha, \mathrm{n}){ }^{16} \mathrm{O}$ reaction. The $\mathrm{H}$-ingestion flash may not cause a split of the convection zone as it was seen in simulations of He-shell flashes in post-AGB and low- $Z$ asymptotic giant branch (AGB) stars. We estimate that for the production of first-peak heavy elements this site can be of similar importance for galactic chemical evolution as the s-process production by low-mass AGB stars. The He-shell flashes result in the expansion and, ultimately, ejection of the accreted and then i-process enriched material, via super-Eddington-luminosity winds or Roche-lobe overflow. The WD models do not retain any significant amount of the accreted mass, with a He retention efficiency of $\lesssim 10 \%$ depending on mass and convective boundary mixing assumptions. This makes the evolutionary path of such systems to supernova Ia explosion highly unlikely.
\end{abstract}

Key words: binaries: close - stars: abundances - stars: evolution - stars: interiors - supernovae: general

\section{Introduction}

Trans-iron elements are produced through n-capture nucleosynthesis, such as the slow (s; with a neutron density $N_{\mathrm{n}} \lesssim 10^{11} \mathrm{~cm}^{-3}$ ) and the rapid ( $\mathrm{r} ; N_{\mathrm{n}} \gtrsim 10^{20} \mathrm{~cm}^{-3}$ ) processes (e.g., Käppeler et al. 2011; Thielemann et al. 2011). Cowan \& Rose (1977) proposed that an i process with $N_{\mathrm{n}} \sim 10^{15} \mathrm{~cm}^{-3}$ intermediate between $\mathrm{s}$ and $\mathrm{r}$ processes might be triggered when $\mathrm{H}$ is mixed into a convective He-burning shell. Neutrons for the i process are released in the reaction ${ }^{13} \mathrm{C}(\alpha, \mathrm{n}){ }^{16} \mathrm{O}$. The high $\mathrm{n}$ density in the i process is achieved at typical He-burning temperatures in the range $2-3 \cdot 10^{8} \mathrm{~K} .{ }^{13} \mathrm{C}$ is rapidly produced by the $\beta^{+}$decay of ${ }^{13} \mathrm{~N}$ with a half-life of 9.96 minutes, which in turn is the result of the reaction ${ }^{12} \mathrm{C}(\mathrm{p}, \gamma){ }^{13} \mathrm{~N}$ that operates here on the convective turnover timescale of $\approx 15$ minutes (Herwig et al. 2011). The He- and H-burning reactions are spatially separated, each occurring at its own favorable conditions, and ${ }^{13} \mathrm{~N}$ decays into ${ }^{13} \mathrm{C}$ while being carried down by convection, thus avoiding its destruction by a further proton capture (e.g., Campbell et al. 2010; Herwig et al. 2011; Lugaro et al. 2012).

The first evidence of i process in stars were observations by Asplund et al. (1999) of $\gtrsim 2$ dex enhancement of the light (firstpeak) s-process elements, such as $\mathrm{Rb}, \mathrm{Sr}$, and $\mathrm{Y}$, but not of the second-peak elements $\mathrm{Ba}$ and $\mathrm{La}$ in the post-AGB very late thermal pulse (VLTP) star Sakurai's object (Herwig 2001; Werner \& Herwig 2006). A VLTP is a He-shell flash in a postAGB star or young white dwarf (WD) that has already entered

\footnotetext{
${ }^{8}$ NuGrid collaboration, http://www.nugridstars.org.
}

the WD cooling track, i.e., the H-burning shell has turned off. He-shell flashes are commonly and recurrently happening in AGB stars (Herwig 2005). In the VLTP, the He-convection zone reaches into the remaining $\mathrm{H}$-rich atmosphere, which will mix protons into He-burning conditions (Fujimoto 1977; Iben 1982). This mixing triggers i-process nucleosynthesis, and simulations of this situation can reproduce the unusual heavy element i-process fingerprint in Sakurai's object if mixing predictions from a mixing-length theory are modified to reflect possible effects of the inhomogeneous nature of convectivereactive burn in H-ingestion events (Herwig et al. 2011, 2014). The isotopic signature of i process has since been identified as well in pre-solar grains (Fujiya et al. 2013; Jadhav et al. 2013; Liu et al. 2014).

Evidence for i-process nucleosynthesis has also been reported in the anomalous $\mathrm{Ba}$ enrichment in some open clusters (Mishenina et al. 2015), while Dardelet et al. (2014) showed that nucleosynthesis in i-process conditions provided a remarkable match to the abundances in several CEMP-r/s (carbon enhanced metal-poor with simultaneous presence of $\mathrm{s}$ elements and $\mathrm{Eu}$ ) stars. Possible sites for the i process include the He-core flash (e.g., Campbell et al. 2010), He-shell flashes in low-Z AGB stars (e.g., Iwamoto et al. 2004) and super-AGB stars (Jones et al. 2016).

Post-AGB VLTPs from a single-star channel are not important sources of heavy elements on a galactic chemical evolution scale. However, Cassisi et al. (1998) reported that $\approx 0.6 M_{\odot}$ WDs that accreted solar-composition material at rates of $4 \cdot 10^{-8}$ and $10^{-7} M_{\odot} \mathrm{yr}^{-1}$ would eventually experience He- 
Table 1

RAWD Model Parameters

\begin{tabular}{|c|c|c|c|c|c|c|c|c|c|}
\hline ID & $\begin{array}{l}M_{\mathrm{WD}} \\
\left(M_{\odot}\right)\end{array}$ & $\begin{array}{l}\dot{M}_{\mathrm{acc}} \\
\left(\frac{M_{\odot}}{\mathrm{yr}}\right)\end{array}$ & $\begin{array}{c}T_{\mathrm{c}} \\
\left(10^{6} \mathrm{~K}\right)\end{array}$ & $f_{\text {bot }}$ & $\begin{array}{c}R_{\mathrm{RL}, \mathrm{WD}} \\
\quad\left(R_{\odot}\right)\end{array}$ & $\begin{array}{l}\text { Mass } \\
\text { Loss }\end{array}$ & $\begin{array}{c}\text { Comp. He } \\
\text { Flashes }\end{array}$ & $\begin{array}{l}L_{\mathrm{He}}^{\max } \\
\left(L_{\odot}\right)\end{array}$ & $\begin{array}{c}\eta_{\mathrm{He}} \\
\%\end{array}$ \\
\hline $\bar{A}$ & 0.65 & $1 \cdot 10^{-7}$ & 21 & 0 & 0.65 & RLOF & 2 & $7.4 \cdot 10^{10}$ & 6.8 \\
\hline B & 0.73 & $2 \cdot 10^{-7}$ & 44 & 0 & 0.64 & RLOF & 6 & $4.4 \cdot 10^{9}$ & 6.1 \\
\hline $\mathrm{C}$ & 0.73 & $2 \cdot 10^{-7}$ & 44 & 0.008 & 2.2 & $L>L_{\text {Edd }}$ & 19 & $6.7 \cdot 10^{9}$ & -3.7 \\
\hline $\mathrm{D}$ & 1.00 & $2 \cdot 10^{-7}$ & 20 & 0.004 & $\mathrm{n} / \mathrm{a}$ & $L>L_{\mathrm{Edd}}$ & 4 & $3.2 \cdot 10^{10}$ & 10 \\
\hline
\end{tabular}

shell flashes with $\mathrm{H}$-ingestion. Their simulations ended due to the ensuing numerical difficulties, but Cassisi et al. (1998) suggested that those He-shell flashes could lead to substantial additional mass loss as they would cause the WD to evolve back toward the giant branch, just as the VLTP model of Sakurai's object.

This raises several questions. What is the effect of recurrent He-shell flashes on the mass retention efficiency and the implication for the single-degenerate supernova of type Ia (SN Ia, Hillebrandt et al. 2013) progenitor channel? What is the nucleosynthesis signature of rapidly accreting WD (RAWD) He-shell flashes with $\mathrm{H}$ ingestion? Do RAWDs contribute to the chemical inventory of the Galaxy. In this paper we are starting to address these questions through simulations of RAWDs for parameters that are similar to those considered for the single-degenerate progenitor evolution channel of SNe Ia. Model assumptions and simulations are presented in Section 2, followed by our results (Section 3) and conclusions (Section 4).

\section{Model Assumptions and Simulations}

As in our previous work on slowly accreting WDs (the Nova Framework; Denissenkov et al. 2013, 2014), we used the MESA stellar evolution code (here, rev. 5329; Paxton et al. 2013) with the same microphysics, except for the reaction network where we included the pp chains, $\mathrm{CNO}, \mathrm{NeNa}$, and $\mathrm{MgAl}$ cycles, as well as the main reactions of He burning. The accreted material and the WD both have the solar initial composition (Grevesse \& Noels 1993; Lodders 2003). Like in our nova models, we have taken into account convective boundary mixing (CBM) at the bottom of the He-flash convective zone in some of the evolution tracks via the exponential, diffusive model (Freytag et al. 1996; Herwig 2000; Battino et al. 2016) implemented in MESA. The efficiency parameter $f_{\text {bot }}$ specifies the e-folding distance of the exponential decay of the mixing efficiency (Table 1).

Binary population synthesis (BPS) predicts the formation of close systems, consisting of a CO WD accretor and a mainsequence (MS) or subgiant donor, suitable for the singledegenerate channel to SNe Ia (e.g., Han \& Podsiadlowski 2004; Chen et al. 2014). For such systems the rate of mass accretion by the WD has to be in a narrow range around $\dot{M}_{\text {acc }} \sim 10^{-7} M_{\odot} \mathrm{yr}^{-1}$ (e.g., Ma et al. 2013; Wolf et al. 2013), so that excessive mass loss due to $\mathrm{H}$-shell nova flashes at lower accretion rates (e.g., Denissenkov et al. 2013) or due to envelope inflation at higher accretion rates is avoided. For our models we adopt $\dot{M}_{\text {acc }} \sim 10^{-7} M_{\odot} \mathrm{yr}^{-1}$.

Next, we have to choose initial values for the WD and donor (secondary) star mass, $M_{\mathrm{WD}}$ and $M_{\mathrm{d}}$, their orbital separation $a$ or period $P_{\text {orb}}$, and the WD's age or central temperature $T_{\mathrm{WD}}$. According to BPS models the most frequent combinations of the WD and donor star masses at the beginning of mass transfer in a binary system from which evolution to $\mathrm{SNe}$ Ia may be possible are $0.5 \lesssim M_{\mathrm{WD}} / M_{\odot} \lesssim 0.7$ and $0.8 \lesssim M_{\mathrm{d}} / M_{\odot} \lesssim 2$, while the suitable orbital periods for the SN Ia outcomes range from 0.4 to 6.3 days, depending on $M_{\mathrm{WD}}$ (Chen et al. 2014).

We calculate rapid accretion on WD models with $M_{\mathrm{WD}} \approx 0.65 M_{\odot}($ model $\mathrm{A})$ and $M_{\mathrm{WD}} \approx 1 M_{\odot}($ model D) from the Nova Framework (Denissenkov et al. 2013). In addition, we construct a new CO WD model with $M_{\mathrm{WD}} \approx 0.73 M_{\odot}$ including the pre-WD evolution starting on the pre-MS (models $B$ and $C$ ). The WD Roche-lobe radii adopted for our simulations are given in Table 1 and correspond to periods of $\sim 0.22$ days for models $\mathrm{A}$ and $\mathrm{B}$ and $\sim 1.4$ days for model $\mathrm{C}$ for a secondary mass $\sim 1 M_{\odot}$.

The WD ages when accretion starts follow from basic properties of the binary evolution scenario, and the corresponding WD central temperatures of our models are of the order of a few $10^{7} \mathrm{~K}$ (Table 1 ). The WD cools down until its MS or subgiant binary companion fills its Roche lobe and mass transfer starts. For model C, an adopted 1.5 $M_{\odot}$ MS donor star would expand to its Roche-lobe radius in $2.426 \mathrm{Gyr}$. It would take $0.252 \mathrm{Gyr}$ for the $3.7 M_{\odot}$ primary to evolve to the beginning of the WD cooling sequence, where the WD has the central temperature $T_{\mathrm{WD}} \approx 10^{8} \mathrm{~K}$. During the remaining 2.174 Gyr between the two times, the WD would cool down to $T_{\mathrm{WD}}<1.0 \cdot 10^{7} \mathrm{~K}$.

Rotation possibly caused by the accretion of angular momentum is not taken into account. Such rotation may weaken He-shell flashes in a massive and hot CO WD (Yoon et al. 2004) and may increase the retention efficiency of accreted material. We consider less massive and cooler $\mathrm{CO}$ WDs that experience much stronger He-shell flashes, as described below. The low mass retention efficiency of our models implies that any accreted angular momentum would be immediately lost again, which would lessen the effect of rotation.

\section{Results}

Models $\mathrm{B}$ and $\mathrm{C}$ are based on the MESA test suite case make_co_wd. A star with initially $3.7 M_{\odot}$ evolves from the pre-MS through the usual evolutionary stages to the asymptotic giant branch (AGB; Figure 1). At that point, the star is forced to lose most of its mass by artificially imposing a very high massloss rate, as if it went through a common-envelope event. As a result, the star leaves the AGB and evolves into a post-AGB star and then pre-WD with $M_{\mathrm{WD}} \approx 0.73 M_{\odot}$. This model happens to experience a VLTP (the loop starting at $\log _{10} T_{\text {eff }} \approx 5.25$ ), just like the evolution track for Sakurai's object. We then let the WD cool down to the adopted core temperature and start the accretion (Table 1) of solarcomposition material. 


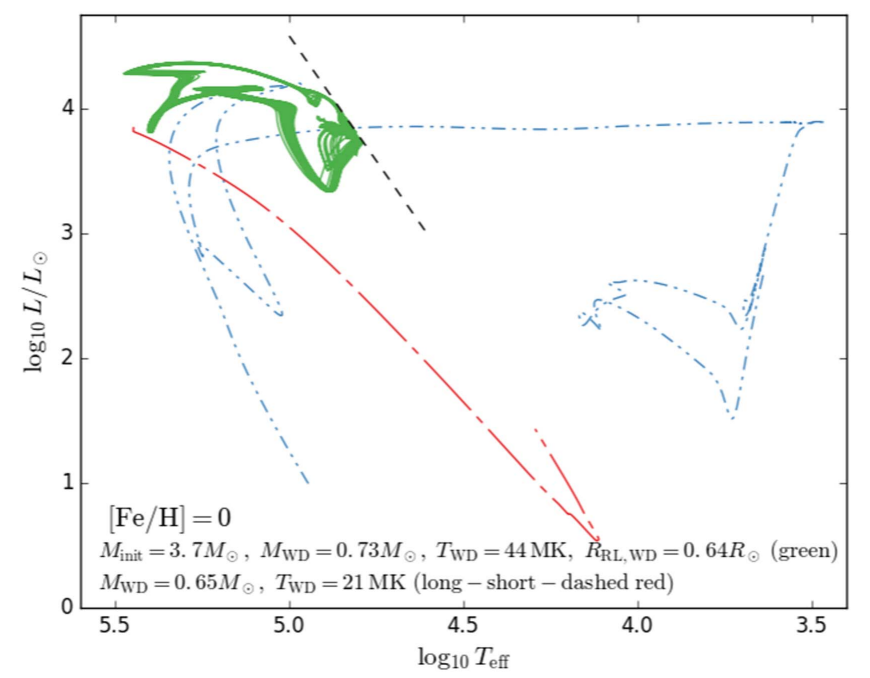

Figure 1. Tracks of progenitor and post-AGB evolution of $0.73 M_{\odot}$ WD (double-dot-short-dashed blue line; see text for details) and multiple He-shell flashes with $\mathrm{H}$-ingestion cycles in model B (green line). The flash causes the star to expand to the WD Roche-lobe radius (dashed black line) and lose the accreted material via the Roche-lobe overflow. The short-long-dashed red curve is a fragment of the track of model A during its second He-shell flash.

\subsection{The Multi-cycle He-flash Evolution and the He Retention Efficiency}

When a sufficiently thick layer of $\mathrm{He}\left(\approx 0.012 M_{\odot}\right.$ for models $\mathrm{B}$ and $\mathrm{C}$ ) is accumulated through steady $\mathrm{H}$-shell burning a convective He-shell flash is triggered (as in model A shown in Figure 3), just as it would be in an AGB stellar evolution sequence (Herwig 2005). The flash leads to the expansion of the simulated star just as in the post-AGB VLTP stellar evolution models (Werner \& Herwig 2006). Mass loss starts as soon as the stellar model exceeds the Eddington luminosity $\left(\dot{M}_{\text {wind }} \approx 10^{-4} M_{\odot} \mathrm{yr}^{-1}\right.$, model C) or when its radius reaches the Roche-lobe radius $\left(\dot{M}_{\text {wind }} \approx 10^{-3} M_{\odot} \mathrm{yr}^{-1}\right.$, model B). Both the accretion and further expansion pause during the mass loss. When a certain amount of mass is lost, the model contracts again and the accretion of solar-composition material resumes. During this duty cycle, the star makes a loop on the HR diagram (Figure 1). In models $\mathrm{C}$ and $\mathrm{D}$ the mass loss is dominated by the super-Eddington-luminosity wind. Yet, the peak He-burning luminosities and temperatures remain quantitatively very similar to those obtained in model B.

One of the most important parameters in BPS studies of the single-degenerate SN Ia progenitor channel is the He retention efficiency, $\eta_{\mathrm{He}}$. It gives a fraction of the He-shell mass $\Delta M_{\mathrm{He}}$ that is left on the WD after the He-shell flash. It has usually been assumed to be $100 \%$. This parameter has, to the best of our knowledge, never been estimated in stellar evolution computations of recurrent He-shell flashes alternating with phases of steady $\mathrm{H}$ burning in RAWDs. The largest $\mathrm{He}$ retention efficiency $\eta_{\mathrm{He}}=10 \%$ is found for the highest-mass model (D) with a very small CBM efficiency, while the lowermass models without $\mathrm{CBM}$ (A and B) show $\eta_{\mathrm{He}} \sim 6 \%$ and the $0.73 M_{\odot}$ model with $\mathrm{CBM}$ has $\eta_{\mathrm{He}}=-3.7 \%$ (Table 1 and Figure 2). In the last case, the mass of the WD decreases with time; therefore, it cannot reach the Chandrasekhar mass, unless for some reason, $\eta_{\mathrm{He}}$ increases later in the accretion evolution. Based on numerous investigations of the effect that CBM at the bottom of the He-shell flash convection zone has on stellar evolution simulations (e.g., Herwig 2005; Miller Bertolami

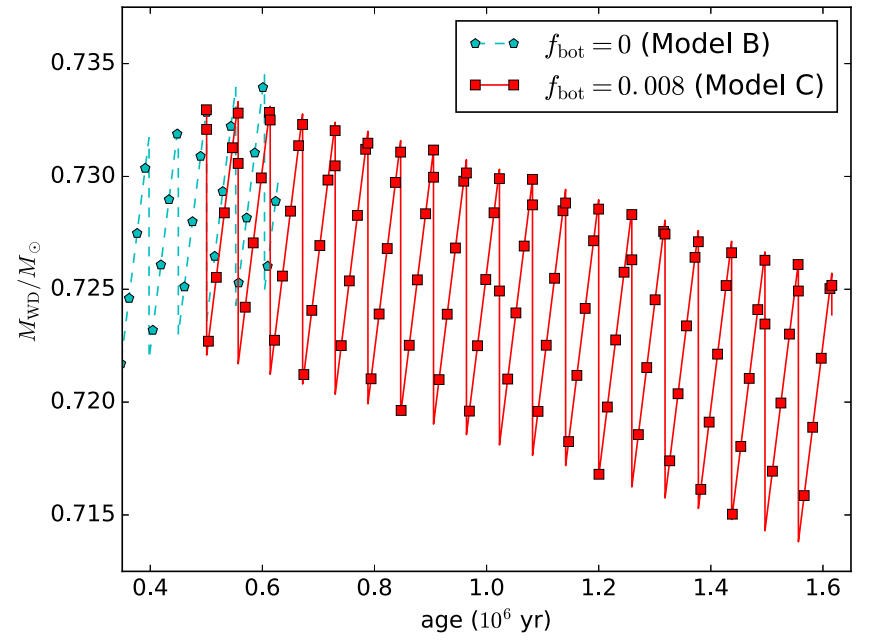

Figure 2. Evolution of the total mass of the $0.73 M_{\odot}$ RAWD model B without CBM $\left(f_{\text {bot }}=0\right)$ and model C with CBM $\left(f_{\text {bot }}=0.008\right)$. Each of the sawtooth-shaped features represents the mass increase during the accretion phase followed by mass ejection in Roche-lobe overflow (model B) or Eddingtonluminosity mass loss (model C) triggered by the He-shell flash.

et al. 2006; Werner \& Herwig 2006; Weiss \& Ferguson 2009; Karakas et al. 2010; Battino et al. 2016), its investigation in 3D simulations (Herwig et al. 2007) and the better agreement of observations of novae with models that include CBM at the bottom of the H-flash convection (Denissenkov et al. 2013), we conclude that CBM with $f_{\text {bot }}=0.008$ at the bottom of the Heshell flash convection zone is at present the most realistic assumption.

\subsection{The i Process}

The He-shell flashes that we encounter in the rapidly accreting white dwarf (RAWD) models are similar to the postAGB He-shell flash model that matches the observations of Sakurai's object, in the sense that also in the RAWD models $\mathrm{H}$ from the envelope $\left(\Delta M_{\text {env }} \sim 10^{-5}\right.$ to $\left.10^{-4} M_{\odot}\right)$ is ingested into the He-shell flash convection (Figure 3). The H-ingestion events are seen in all of our simulated He-shell flashes.

The ingested protons react with ${ }^{12} \mathrm{C}$ to form ${ }^{13} \mathrm{~N}$. Over its $\beta^{+}$-decay timescale it is advected toward the bottom of the Heshell flash convection zone, where temperatures are high enough to activate a rapid release of neutrons via the ${ }^{13} \mathrm{C}(\alpha, \mathrm{n}){ }^{16} \mathrm{O}$ reaction. Just as in the case of Sakurai's object (Herwig et al. 2011), H-ingestion events in RAWD models lead to i-process nucleosynthetic abundance patterns. Given that most or all of the material from the He-shell convective zone is ejected (Section 3.1), RAWDs may be important sources of enrichment of the ISM in heavy elements produced in the $\mathrm{i}$ process (Section 4).

One important difference between the H-ingestion RAWD models and the VLTP or low-Z AGB models is a result of the WD age and associated compactness. This causes He-shell flashes in RAWDs to be very strong. The peak He-burning luminosity is $L_{\mathrm{He} \text {,peak }}=10.869$ and remains for most of the $\mathrm{H}$-ingestion event much higher than the energy released by $\mathrm{H}$ burning that reaches $L_{\mathrm{H} \text {,peak }}=9.673$ (Figure 3 ). In spite of this enormous energy input into the He-shell flash convection zone from the burning of ingested $\mathrm{H}$, the stellar evolution simulation does not indicate the formation of a split of the convection zone and the formation of a separate H-burning driven convection as 

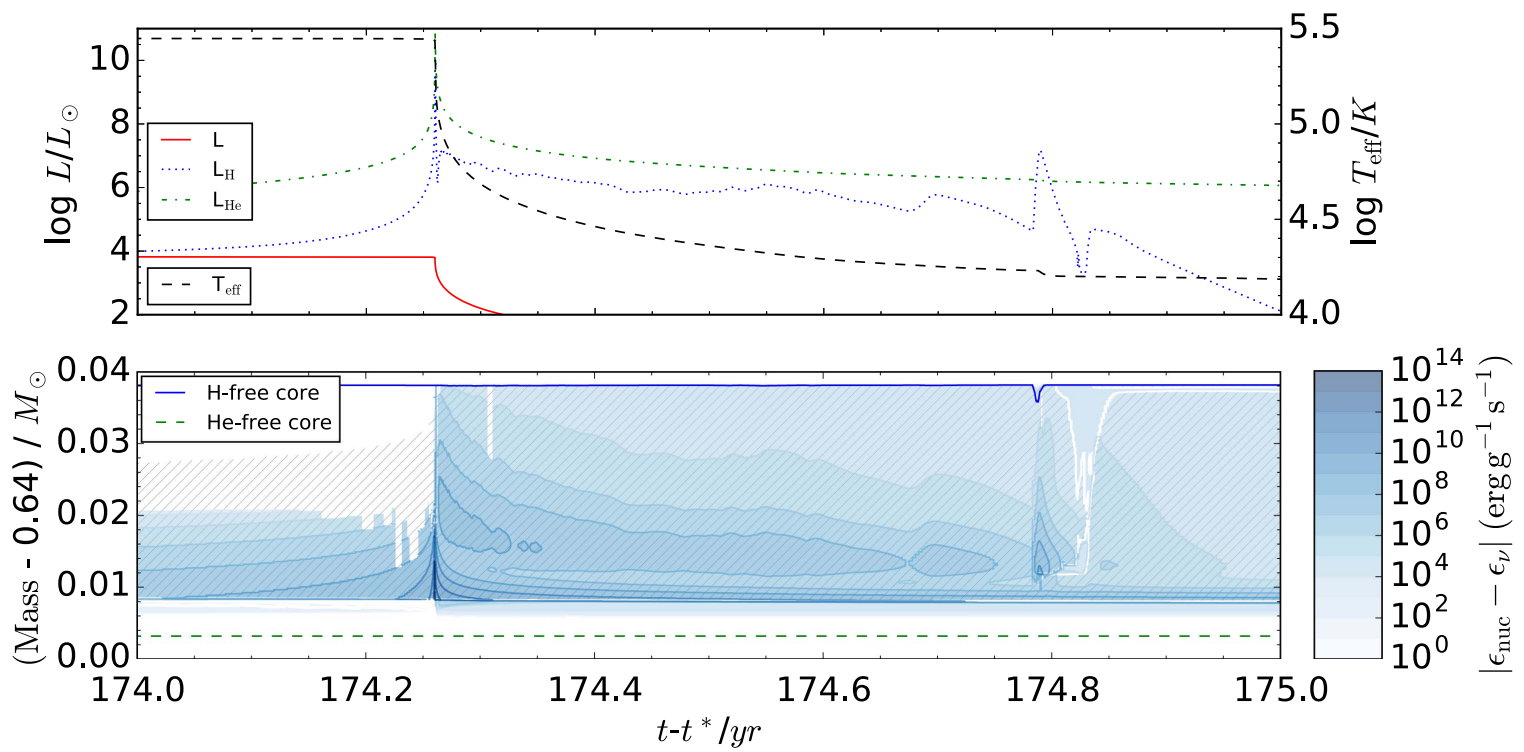

Figure 3. Top panel: $T_{\text {eff }}$ and luminosity evolution (total, and due to $\mathrm{H}$ and He burning separately). Bottom panel: time evolution of convection zone (hatched area), energy generation (gray shades), and $\mathrm{H}$ - and $\mathrm{He}$-free mass coordinates diagram of the He-shell flash in model A. $t_{*}$ corresponds to the onset of the flash.

has been commonly observed in H-ingestion simulations of post-AGB and low-Z AGB stars (Section 1).

During the initial phase of the He-shell flash of model A at $t=174.25$ years, we first observe a very high $\mathrm{H}$-ingestion rate followed by a prolonged period of lower $\mathrm{H}$ ingestion, and all of this without split of the He-flash convection zone. If it is confirmed in 3D simulations that no global oscillation of shell H-ingestion (GOSH; Herwig et al. 2014) is found in this situation, 1D spherically symmetric simulations of the i-process nucleosynthesis in RAWDs are probably more realistic compared to the case of post-AGB VLTP models and low- $Z$ AGB models for which 3D stellar hydrodynamics simulations have yet to determine the detailed outcome of the GOSH.

At a later time ( $t=174.8$ years), the $\mathrm{H}$-ingestion rate does increase for a brief period when the convection zone is indeed split. Whether or not this particular event is due to the decreasing He-burning luminosity, and how the detailed properties of this event depend on numerical and physics model assumptions is not yet clear. However, our simulations suggest that there is a distinct possibility of diversity in the convective mixing and nuclear burning interactions in $\mathrm{H}$-ingestion events on degenerate cores.

We adopt the same 1D multi-zone post-processing nucleosynthesis simulation approach as in Herwig et al. (2011, for details see Section 5.1 there) using the NuGrid MPPNP code (Pignatari et al. 2016). The initial abundances for the simulations are taken from Asplund et al. (2005) with isotopic ratios from Lodders (2003). The initial abundances of some light intershell species, such as ${ }^{4} \mathrm{He},{ }^{12} \mathrm{C},{ }^{16} \mathrm{O}$ are modified to reflect their intershell abundance due to the progenitor AGB evolution. The MPPNP code dynamically includes up to 5234 isotopes as needed and the associated reaction rates from JINA REACLIB V1.1 (Cyburt et al. 2010) and select other sources (Pignatari et al. 2016).

We perform simulations for an initial prolonged ingestion $(\sim 0.38$ year $)$ without split with a low ingestion rate $\left(\sim 1.8 \cdot 10^{-12} M_{\odot} \mathrm{s}^{-1}\right)$ as well as the subsequent shorter event $(\sim 0.0084$ year $)$ with a high ingestion rate $\left(\sim 8.9 \cdot 10^{-11} M_{\odot} \mathrm{s}^{-1}\right)$ that does lead to a convection zone split. The $T-\rho$ profile of the He-shell convective zone was taken

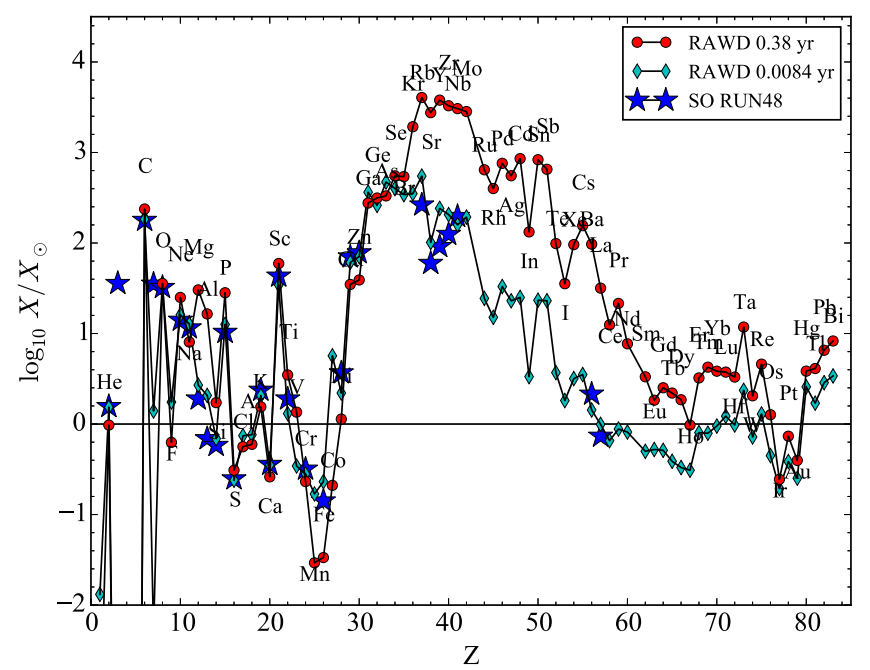

Figure 4. Abundance distributions in the RAWD model A, after the second $\mathrm{He}$ flash, with $M_{\mathrm{WD}}=0.65 M_{\odot}$ and $T_{\mathrm{WD}}=21 \mathrm{MK}$ (teal diamonds, solid lines), and for comparison the model RUN48 (blue stars) from Herwig et al. (2011) that matches the observed abundances of Sakurai's object.

from model A when the H-ingestion has just begun during the second He-shell flash.

In both simulations, a substantial overproduction of mostly first-peak trans-iron elements is found (Figure 4). The abundance signature of the short event (H-ingestion with split) is very similar to the post-AGB He-shell flash simulation that matches the observed abundance distribution in Sakurai's object. The longer event (without split) shows even higher overproduction up to 3.5 dex and the abundance distribution is starting to spill over the first-peak $N=50$ magic neutron number, generating elements such as Mo and $\mathrm{Ag}$. These simulations demonstrate that the ejecta of RAWDs may be enriched with first-peak heavy elements by $2.5-3.5$ dex, and that a range of conditions may lead to a certain diversity of local elemental ratios within the i-process paradigm. 


\section{Conclusion}

Iben (1981) proposed that accreting WDs may be a potential astrophysical site for the sprocess, via the ${ }^{22} \mathrm{Ne}(\alpha, \mathrm{n})^{25} \mathrm{Mg}$ reaction in He-shell flashes. It was later realized that the main $\mathrm{s}$ process is best associated with the radiative ${ }^{13} \mathrm{C}$ neutron source (Gallino et al. 1998) in AGB stars. Our models show that for WD accretion models with $M_{\mathrm{WD}} \sim 0.7-0.8 M_{\odot}$ the nucleosynthesis signature should instead be dominated by the i process fueled by the convective ${ }^{13} \mathrm{C}(\alpha, \mathrm{n}){ }^{16} \mathrm{O}$ neutron source.

The Milky Way present-day star formation rate is $\sim 2 M_{\odot} \mathrm{yr}^{-1}$ (Chen et al. 2014). A fraction of $\sim 0.08$ of this will go into low-mass AGB stars $\left(1.5 M_{\odot} \leqslant M_{\text {ini }} \leqslant 3 M_{\odot}\right)$ that produce the main s process, and they return $\sim 70 \%$ of their mass to the ISM. Therefore, $\approx 0.1 M_{\odot} \mathrm{yr}^{-1}$ of s-process enriched material is returned by low-mass AGB stars. This material is enriched by $\sim 2$ compared to the initial abundance of heavy elements (e.g., Lugaro et al. 2003).

As a lower limit, we adopt for the RAWD rate the presently estimated rate of SNe Ia from the single-degenerate channel, $2 \times 10^{-4}$ year $^{-1}$ (Chen et al. 2014). If one assumes that for each RAWD $\sim 0.5 M_{\odot}$ of H-rich material can be accreted, and ejected enriched with i-process elements (assuming $\eta_{\mathrm{He}}=0$ ), then $\approx 0.0001 M_{\odot} \mathrm{yr}^{-1}$ of i-process enriched material is returned by RAWDs with an enrichment factor of $\sim 1000$ (Figure 4).

The ratio of the contributions of elements made by both lowmass AGB stars and RAWDs is then

$$
\frac{\mathrm{AGB} \text { contribution }}{\text { RAWD contribution }}=\frac{0.1 M_{\odot} \mathrm{yr}^{-1}}{0.0001 M_{\odot} \mathrm{yr}^{-1}} \times \frac{2}{1000}=2.0 \text {. }
$$

Therefore, based on results shown in Figure 4, we propose that the i process in RAWDs may be a relevant astrophysics source for elements in the Ge-Mo region. The i process in RAWDs could be one of the nucleosynthesis components explaining the missing solar LEPP (Travaglio et al. 2004; Montes et al. 2007).

Another important result is that, according to our stellar evolution models of RAWDs that follow through their He-shell flashes, the He retention efficiencies are $\lesssim 10 \%$, or negative in models with CBM. This result is consistent with the recent Heaccreting WD models (Wang et al. 2015 and references therein). BPS estimates of the SN Ia rate from the singledegenerate channel usually assume a He retention efficiency of $100 \%$ (e.g., Chen et al. 2014). This means that according to our models this channel for SNe Ia via RAWDs is highly unlikely, except possibly for a very small fraction of systems with the most massive WDs and donor stars. RAWD systems are predicted by numerous population synthesis models. They should appear as super-soft X-ray sources for the most time, unless being (easily) obscured by interstellar or circum-binary matter (van den Heuvel et al. 1992). The latter factor probably explains why only a few RAWD candidates out of theoretically predicted dozens were found in the LMC and SMC (Lepo \& van Kerkwijk 2013).

This material is based upon work supported by the National Science Foundation under grant No. PHY-1430152 (JINA Center for the Evolution of the Elements). F.H. acknowledges funding from an NSERC Discovery grant. M.P. acknowledges the support from SNF (Switzerland) and from the "Lendulet-2014" Programme of the Hungarian Academy of Sciences (Hungary). S. $\mathrm{J}$. is a fellow of the Alexander von Humboldt Foundation and acknowledges support from the Klaus Tschira Foundation.

\section{References}

Asplund, M., Grevesse, N., \& Sauval, A. J. 2005, in ASP Conf. Ser. 336, Cosmic Abundances as Records of Stellar Evolution and Nucleosynthesis, ed. T. G. Barnes, III \& F. N. Bash (San Francisco, CA: ASP), 25

Asplund, M., Lambert, D. L., Kipper, T., Pollacco, D., \& Shetrone, M. D. 1999, A\&A, 343, 507

Battino, U., Pignatari, M., Ritter, C., et al. 2016, ApJ, 827, 30

Campbell, S. W., Lugaro, M., \& Karakas, A. I. 2010, A\&A, 522, L6

Cassisi, S., Iben, I. J., \& Tornambé, A. 1998, ApJ, 496, 376

Chen, H.-L., Woods, T. E., Yungelson, L. R., Gilfanov, M., \& Han, Z. 2014, MNRAS, 445, 1912

Cowan, J. J., \& Rose, W. K. 1977, ApJ, 212, 149

Cyburt, R. H., Amthor, A. M., Ferguson, R., et al. 2010, ApJS, 189, 240

Dardelet, L., Ritter, C., Prado, P., et al. 2014, in Proceedings of Science (NIC XIII), ed. Z. Elekes \& Z. Fülöp (Trieste: SISSA), 145

Denissenkov, P. A., Herwig, F., Bildsten, L., \& Paxton, B. 2013, ApJ, 762,8

Denissenkov, P. A., Truran, J. W., Pignatari, M., et al. 2014, MNRAS, 442, 2058

Freytag, B., Ludwig, H.-G., \& Steffen, M. 1996, A\&A, 313, 497

Fujimoto, M. Y. 1977, PASJ, 29, 331

Fujiya, W., Hoppe, P., Zinner, E., Pignatari, M., \& Herwig, F. 2013, ApJL, 776, L29

Gallino, R., Arlandini, C., Busso, M., et al. 1998, ApJ, 497, 388

Grevesse, N., \& Noels, A. 1993, in Origin and Evolution of the Elements, ed. N. Prantzos, E. Vangioni-Flam, \& M. Casse (Cambridge: Cambridge Univ. Press), 15

Han, Z., \& Podsiadlowski, P. 2004, MNRAS, 350, 1301

Herwig, F. 2000, A\&A, 360, 952

Herwig, F. 2001, ApJL, 554, L71

Herwig, F. 2005, ARA\&A, 43, 435

Herwig, F., Freytag, B., Fuchs, T., et al. 2007, in ASP Conf. Ser. 378, Why Galaxies Care About AGB Stars: Their Importance as Actors and Probes, ed. F. Kerschbaum, C. Charbonnel, \& R. F. Wing (San Francisco, CA: ASP), 43

Herwig, F., Pignatari, M., Woodward, P. R., et al. 2011, ApJ, 727, 89

Herwig, F., Woodward, P. R., Lin, P.-H., Knox, M., \& Fryer, C. 2014, ApJL, 792, L3

Hillebrandt, W., Kromer, M., Röpke, F. K., \& Ruiter, A. J. 2013, FrPhy, 8,116

Iben, I. J. 1982, ApJ, 260, 821

Iben, I., Jr. 1981, ApJ, 243, 987

Iwamoto, N., Kajino, T., Mathews, G. J., Fujimoto, M. Y., \& Aoki, W. 2004, ApJ, 602, 377

Jadhav, M., Pignatari, M., Herwig, F., et al. 2013, ApJL, 777, L27

Jones, S., Ritter, C., Herwig, F., et al. 2016, MNRAS, 455, 3848

Käppeler, F., Gallino, R., Bisterzo, S., \& Aoki, W. 2011, RvMP, 83, 157

Karakas, A. I., Campbell, S. W., \& Stancliffe, R. J. 2010, ApJ, 713, 374

Lepo, K., \& van Kerkwijk, M. 2013, ApJ, 771, 13

Liu, N., Savina, M. R., Davis, A. M., et al. 2014, ApJ, 786, 66

Lodders, K. 2003, ApJ, 591, 1220

Lugaro, M., Herwig, F., Lattanzio, J. C., Gallino, R., \& Straniero, O. 2003, ApJ, 586, 1305

Lugaro, M., Karakas, A. I., Stancliffe, R. J., \& Rijs, C. 2012, in AIP Conf. Ser. 1484, Origin of Matter and Evolution of Galaxies 2011, ed. S. Kubono et al. (Melville, NY: AIP), 111

Ma, X., Chen, X., Chen, H.-1., Denissenkov, P. A., \& Han, Z. 2013, ApJL, 778, L32

Miller Bertolami, M. M., Althaus, L. G., Serenelli, A. M., \& Panei, J. A. 2006, A\&A, 449, 313

Mishenina, T., Pignatari, M., Carraro, G., et al. 2015, MNRAS, 446, 3651

Montes, F., Beers, T. C., Cowan, J., et al. 2007, ApJ, 671, 1685

Paxton, B., Cantiello, M., Arras, P., et al. 2013, ApJS, 208, 4

Pignatari, M., Herwig, F., Hirschi, R., et al. 2016, ApJS, 225, 24

Thielemann, F.-K., Hirschi, R., Liebendörfer, M., \& Diehl, R. 2011, in Lecture Notes in Physics, Vol. 812, ed. R. Diehl, D. H. Hartmann, \& N. Prantzos (Berlin: Springer)

Travaglio, C., Gallino, R., Arnone, E., et al. 2004, ApJ, 601, 864

van den Heuvel, E. P. J., Bhattacharya, D., Nomoto, K., \& Rappaport, S. A. 1992, A\&A, 262, 97

Wang, B., Li, Y., Ma, X., et al. 2015, A\&A, 584, 37

Weiss, A., \& Ferguson, J. W. 2009, A\&A, 508, 1343

Werner, K., \& Herwig, F. 2006, PASP, 118, 183

Wolf, W. M., Bildsten, L., Brooks, J., \& Paxton, B. 2013, ApJ, 777, 136

Yoon, S.-C., Langer, N., \& Scheithauer, S. 2004, A\&A, 425, 217 